\title{
Análisis florístico de las escombreras del centro-norte de la provincia de Valladolid (España)
}

\author{
Jesús Antonio Lázaro Bello (*)
}

\begin{abstract}
Resumen: Lázaro Bello, J.A. Análisis florístico de escombreras situadas en el centro-norte de la provincia de Valladolid (España). Lazaroa 32: 117-136 (2011)

Se aporta un análisis de diversidad florística realizado en dieciocho escombreras localizadas en el centro-norte de la provincia de Valladolid (España). El listado de flora vascular, silvestre y naturalizada, consta de 336 especies pertenecientes a 45 familias diferentes. Se han realizado los espectros taxonómicos, biológicos y corológicos correspondientes. Según el espectro taxonómico Compositae (22,62\%), Gramineae (12,50\%), Leguminosae (8,93\%) y Cruciferae (6,25\%) son las familias que presentan un mayor número de especies. Los terófitos $(50,89 \%)$ y los hemicriptófitos $(30,95 \%)$ son los biotipos mejor representados, y, desde el punto de vista corológico, es preciso destacar el elemento mediterráneo $(52,08 \%)$, con un pequeño porcentaje de endemismos ibéricos $(3,57 \%)$. Además, se han definido dos grupos de escombreras: uno sobre suelos arcillosos y/o margosos (ocho escombreras), y otro sobre suelos calcáreos (diez escombreras), pero no se han observado diferencias estadísticas significativas, al nivel de confianza del 95\%, entre las medias y las desviaciones estándar de ellas.
\end{abstract}

Palabras clave: Escombreras, Diversidad florística, Plantas vasculares, Valladolid, España.

Abstract: Lázaro Bello, J.A. Floristic analysis of dumps located in central-northern Valladolid province (Spain). Lazaroa 32: $117-136(2011)$

The floristic diversity of eighteen dumps located in central-northern Valladolid province (Spain) was analysed. The checklist of natural and subspontaneous vascular plants consists of 336 species belonging to 45 different families. Taxonomic, biological and chorological spectra have been developed. In accordance with the taxonomic spectrum Compositae $(22.62 \%)$, Gramineae $(12.50)$, Leguminosae $(8.93 \%)$ and Cruciferae $(6.25 \%)$ are the families with the largest number of species. Therophytes $(50.89 \%)$ and hemicryptophytes $(30.95 \%)$ are the most represented biological types, and from a chorological standpoint it is necessary to highlight the Mediterranean component (52.08\%) with a short percentage of Iberian endemics (3.57\%). Two groups of dumps have been defined: the first one on clay and/or marl soils (eight dumps), and the second one on calcareous soils (ten dumps), but there is not a statistically significance difference between the means and standard deviations of the two samples at the $95 \%$ confidence level.

Keywords: Dumps, Floristic Diversity, Vascular Plants, Valladolid, Spain.

\section{INTRODUCCIÓN}

En zonas más o menos naturales pero cercanas a núcleos urbanos de población, es frecuente encontrarnos con vertidos variados, como escombros, o residuos orgánicos, arrojados por particulares o empresas que producen un gran impacto visual sobre el paisaje. En función del predominio del tipo de residuo se utilizan vocablos como escombreras o basureros, aunque quizás, el más adecuado sea el de vertederos, como término que agrupa a aquellos sitios o parajes en los que se vierte cualquier tipo de desecho. A pesar de ello vamos a utilizar en este trabajo el de escombreras, ya que la finalidad de este trabajo es el estudio de una flora muy particular, la ruderal, ligada a la presencia de ruinas o escombros (FonT QuER, 2000: 957), sitios que no están preparados, ni acondicionados para actuar como tales, pero que se utilizan con ese fin. Se pueden encontrar en montes públicos, cercanos a cursos fluviales, en márgenes de carreteras y caminos, en vías pecuarias, en solares urbanos, etc. Su presencia determina la aparición de un sustrato nuevo sobre los preexistentes, generándose un nuevo es-

\footnotetext{
* C/ Madre de Dios n ${ }^{\circ}$ 15, $1^{\circ}$ D. E-47011-Valladolid (España). E-mail: chuchijalb@hotmail.com
} 
cenario que permite la invasión por parte de una flora, normalmente, con apetencias por elevadas proporciones de nitrógeno en el medio, removidos y bien oxigenados. Los vertederos que se producen por vertidos sin control alguno suelen albergar residuos no peligrosos o inertes, pero poseen un aspecto desagradable, sin embargo no se ha evaluado adecuadamente la importancia que pueden tener para la supervivencia de algunas especies de flora y fauna silvestre (HERNÁNDEZ LÓPEZ, 1997; SÁNCHEZ \& FERNÁNDEZ, 1988). Su incidencia principal es la degradación del paisaje y la ocupación de suelos, pero en ellos, ciertos taxones, con el paso de tiempo, se han convertido en un verdadero componente de la flora local (TRISKA, 1987: 185), y en algunos casos se les atribuye cierta importancia regional (DEVESA, 1995: 109).

Los herbazales de lugares nitrificados se caracterizan por su alta diversidad florística, con predominio de elementos graminoides o de compuestas espiniscentes que determinan fisionómicamente a los cardales (PenAs \& al., 1987-88; DeVESA, 1995). Estudios fitosociológicos realizados fundamentalmente en provincias vecinas, pero con algún inventario levantado en la provincia vallisoletana (LADERA \& al., 1983; PENAS \& al., 1987-88), incluyen a comunidades vegetales de carácter nitrófilo o subnitrófilo, que pueblan escombreras y con fenología estival, con importante presencia de taxones de los géneros Carduus, Cirsium y Onopordum, pero también de otros como Reseda, Lactuca, etc. Desde el punto de vista biogeográfico, estas comunidades se incluyen en el piso supramediterráneo, sector Castellano-Duriense de la provincia Castellano-Maestrazgo-Manchega (RIVAS-MARTíNEZ \& al., 2002), y son comunes para todo el centro-oeste español (LADERO \& al., 1987-88).

Objetivos de este estudio son aumentar el conocimiento de la composición florística de estos medios en los Montes de Torozos, aportando información corológica de especies que, por comunes, muchas veces no son objeto de demasiada atención. Además, los análisis taxonómicos, biológicos y corológicos servirán para complementar trabajos previos realizados en el mismo tipo de emplazamiento geomorfológico (LÁZARO BELLO, 2005; 2006a), o enfocados al estudio de especies típicas de estos enclaves (LÁZARO BELLO, 2009a). Por otra parte, también pretendemos averiguar si hay diferencias significativas en la composición florística de las escombreras derivadas de las diferencias altitudinales (que son muy pequeñas), y sobre todo litológicas o edafológicas del entorno en que se hallan.

Por último, queremos resaltar la elaboración de un Plan Regional de Residuos Urbanos con el objetivo de desarrollar distintas medidas que culminaron en la Estrategia Regional de Residuos de Castilla y León 2000-2010 (JunTA DE CASTILLA Y LEÓN, 2001: 4). La mencionada estrategia engloba a todos y cada uno de los residuos dentro del alcance y ámbito de aplicación de la Ley 10/1998, de 21 de abril, de Residuos, y tiene, entre sus objetivos cuantitativos, para el caso de los restos de construcción y demolición, la recogida controlada y correcta gestión ambiental de entre el 87 y 100 $\%$ de ellos, y para el caso de los residuos urbanos, la eliminación y sellado de entre el 20 y el $40 \%$ de vertederos (JunTA DE CASTILlA y LeÓN, 2000: 5). A escala provincial, en los últimos años, se están llevando a cabo programas de repoblación forestal y recuperación de escombreras para solventar el elemento disgregador del paisaje agrario en diversos municipios (DiPUTACIÓN DE VALLADOLID, 2010), con la influencia que el desarrollo de dicha estrategia va a tener en las especies que se van a tratar a continuación, en años venideros.

\section{MATERIALES Y MÉTODOS}

\section{ÁREA DE ESTUDIO}

La provincia de Valladolid (España) se encuentra situada en la zona central de la cuenca sedimentaria del Duero. Los materiales fueron depositados en un ambiente continental durante el Terciario y el Cuaternario, siendo el Neógeno el que alcanza mayor desarrollo. La morfología de la zona está relacionada con la horizontalidad o subhorizontalidad de dicho materiales, básicamente miocénicos, y con el diferente grado de competencia entre ellos, lo que da como resultado un modelado diferencial del relieve y la aparición de los tres dominios geomorfológicos clásicos del mioceno castellano: páramos, cuestas y campiñas (HERnÁNDEZ PACHECO, 1915). 
A grandes rasgos, desde el punto de vista climatológico, la provincia posee un marcado clima continental, de influencia atlántica, cuya principal característica es la fuerte oscilación térmica, tanto diaria como anual, con inviernos largos y rigurosos, y veranos cortos, secos y calurosos (GARCíA FERNÁNDEZ, 1986).

Se han estudiado dieciocho escombreras localizadas en la zona centro-norte de la provincia de Valladolid (Figura 1) que ocupan emplazamientos situados en las comarcas de Páramos de Torozos y Páramos de Cerrato o en sus márgenes. La relación de los datos geográficos y altitudinales de las escombreras, así como la fecha en que se llevó a cabo el estudio y el número de especies encontradas, colocados por orden cronológico, fue el siguiente:

1.- Zaratán, 30TUM5113, 760 m, 13-Junio-2009, $\mathrm{n}=136$.

2.- Zaratán, 30TUM4812, 840 m, 14-Junio-2009, $\mathrm{n}=67$.

3.- Wamba, 30TUM4116, 780 m, 14-Junio-2009, $\mathrm{n}=100$.

4.- Cigales, 30TUM5729, 840 m, 19-Junio-2009, $\mathrm{n}=121$.

5.- Ciguñuela, 30TUM4611, 840 m, 20-Junio$2009, \mathrm{n}=95$.

6.- Robladillo, 30TUM4208, 840 m, 20-Junio$2009, \mathrm{n}=89$.
7.- Geria, 30TUM4307, 820 m, 20-Junio-2009, $\mathrm{n}=70$.

8.- Villalba de los Alcores, 30TUM4537, $840 \mathrm{~m}$, 21-Junio-2009, $\mathrm{n}=112$.

9.- Montealegre, 30TUM4441, 810 m, 21-Junio2009, $n=116$.

10.- Corcos del Valle, 30TUM5931, 850 m, 26Junio-2009, n=109.

11.- Cabezón de Pisuerga, 30TUM6722, 840-850 m, 27-Junio-2009, $\mathrm{n}=109$.

12.- Mucientes, 30TUM5422, 760 m, 28-Junio$2009, \mathrm{n}=75$.

13.- Fuensaldaña, 30TUM5418, 760 m, 28-Junio$2009, \mathrm{n}=69$.

14.- Peñaflor de Hornija, 30TUM3520, 840 m, 1Julio-2009, $\mathrm{n}=113$.

15.- La Mudarra, 30TUM3726, 840 m, 1-Julio2009, $n=75$.

16.- San Martín de Valvení, 30TUM7025, 780 m, 2-Julio-2009, $\mathrm{n}=61$.

17.- Cubillas de Santa Marta, 30TUM6432, 800 $\mathrm{m}$, 2-Julio-2009, $\mathrm{n}=68$.

18.- Quintanilla de Trigueros, 30TUM6236, 840 m, 2-Julio-2009, $\mathrm{n}=73$.

Los vertidos de los lugares estudiados están constituidos por restos de materiales de construcción, acompañados en la mayoría de los casos por basuras de diversa índole, y en alguna ocasión

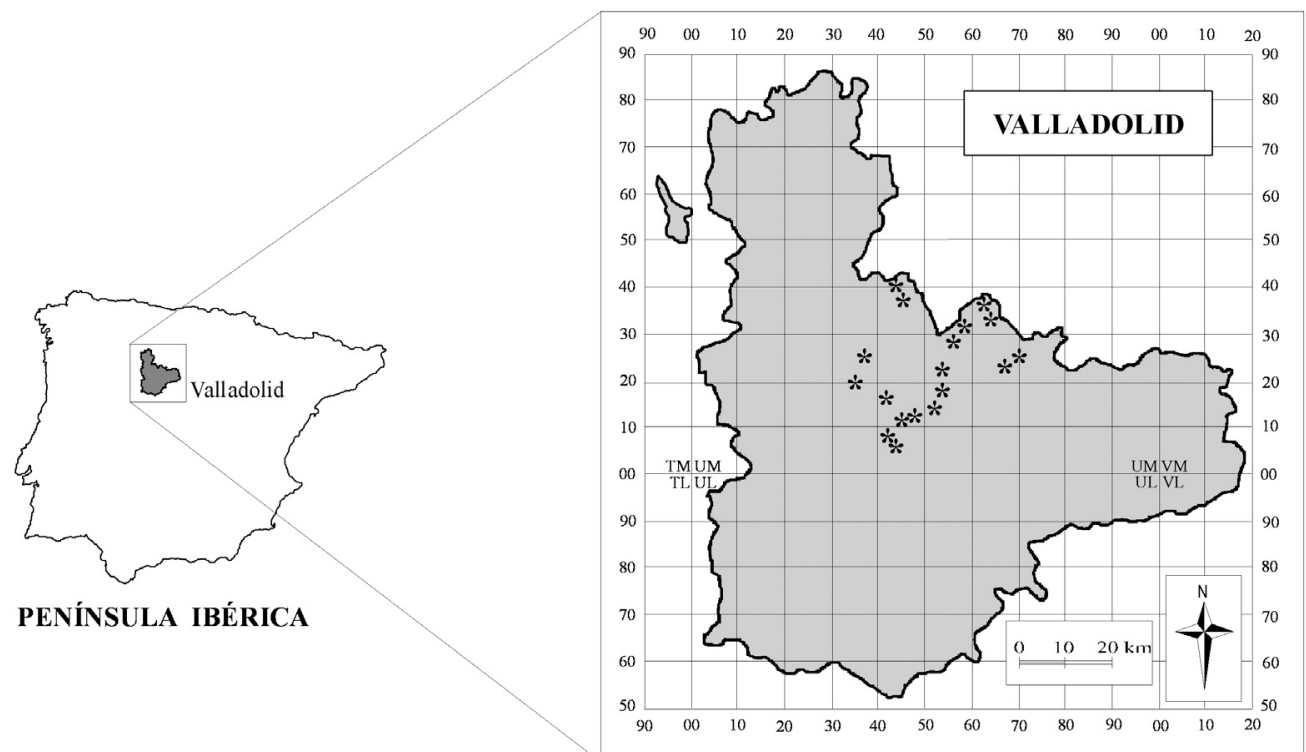

Figure 1. - Mapa de situación y emplazamiento de las escombreras estudiadas. 
con terrenos vecinos muy pastoreados (apriscos, cordeles, etc.). Aunque no se conoce la antigüedad de las escombreras, parece que la media es superior a diez años, o incluso veinte, lo que ha favorecido que muchos de los montículos estén parcialmente recubiertos por sustrato de la zona, por arrastre gracias a la acción eólica. Esto ha permitido, en algunos casos, el asentamiento de especies no propias de escombreras, sino características del entorno inmediato.

\section{ESTUDIO FLORÍSTICO}

Los datos referidos a los inventarios que se levantaron aparecen reflejados en el Anexo 1, y en él los taxones se muestran en forma de listado colocado por orden alfabético de familia, género y especie, señalándose la autoría, el biotipo y la distribución geográfica del citado taxón, así como el número de la escombrera (señalado en el apartado anterior) en que han aparecido.

Aunque el tratamiento que se lleva a cabo durante este estudio considera a los taxones a nivel de especie, el dato que se ha utilizado, cuando procedía, era el de la subespecie inventariada. En los casos en que se han encontrado dos subespecies, se ha tenido en cuenta la distribución geográfica de ambas en conjunto. Esto ha ocurrido con Dactylis glomerata (subsp. glomerata y subsp. hispanica), Galium aparine (subsp. aparine y subsp. spurium), Hordeum murinum (subsp. murinum y subsp. leporinum), Leontodon taraxacoides (subsp. taraxacoides y subsp. hispidus) y Sonchus asper (subsp. asper y subsp. glaucescens).

Con los datos recogidos en los inventarios se ha realizado un análisis taxonómico, biológico y corológico. La nomenclatura botánica adoptada es la que se contempla en Flora iberica (CASTROVIEJO \& al., 1986-2010), y, en su defecto, la adoptada en Flora europaea (TuTin \& al. 1964-1980, 1993), en AizPURU \& al. (1999) o en MATEO SANZ \& CRESPO (2009). Las especies de difícil identificación en el campo se trasladaron al laboratorio para su posterior determinación. A efectos de recuento, debido al ámbito en el que nos hallamos, en el trabajo se presta especial atención al elemento mediterráneo. El resto, elemento eurosiberiano de amplia distribución (plurirregional, subcosmopolita, neófito), se entienden en sentido amplio, de la misma manera en que se recogen en otras grandes obras de uso habitual (AIZPURU \& al., 1999; BolÒs \& VIGO, 1984-2001; VILLAR \& al., 1997-2001), y que ya pusimos de manifiesto en un trabajo anterior (LÁZARo BeLLo, 2006b). Sólo se consideraron las especies reconocibles, normalmente en pleno desarrollo vital, en período de floración o de fructificación. Además, los inventarios se realizaron en un intervalo de menos de tres semanas para evitar grandes diferencias fenológicas. Pliegos testigo de algunos de los taxones citados se hallan recogidos en el herbario del Real Jardín Botánico de Madrid (MA), o en el herbario personal del autor. Además, determinados taxones, dada su importancia a nivel provincial, se han recogido en otras publicaciones (LÁzARo Bello, 2009).

Con la información recogida se han llevado a cabo dos tipos de tratamientos:

a) Estudio en conjunto de todos los inventarios (18 escombreras).

b) Diferenciación de las escombreras en dos grupos basados en diferencias altitudinales y litológicas: un grupo de 8 inventarios (invs. $1,3,7,9,12,13,16,17)$, situados a 760-820 $\mathrm{m}$, sobre sustratos arcillosos y/o margosos (a veces algo yesíferos), y otro grupo de 10 inventarios (invs. 2, 4, 5, 6, 8, 10, 11, 14, 15 , 18 ), situados a $840-850 \mathrm{~m}$, sobre sustrato calcáreo (a veces margo-calcáreo).

\section{ANÁLISIS ESTADÍSTICO DE DATOS}

En todos los casos se comprobó la normalidad y la homocedasticidad, tanto si el índice en estudio era el número de especies, como los porcentajes de biotipos o los de los elementos corológicos. Como estadígrafos de contraste se utilizaron la $\mathrm{F}$ de Snedecor para un ANOVA y el valor del estadístico de Bartlett. La realización de todos los análisis estadísticos y gráficas se llevó a cabo con la utilización de los programas Statgraphics y Excel.

Para el análisis de las familias mejor representadas, se ha procedido al uso del diagrama de caja y patillas, especialmente útil cuando se desea comparar dos o más conjuntos de datos (MiLTON, 1994: 44), y nos ofrece información visual de su 
distribución, la simetría de la misma y su rango (con los valores mínimo, máximo, cuartil inferior y cuartil superior), así como los valores atípicos (outliers). En dicho diagrama, para los valores observados en las muestras, se abunda en el hecho de no percibir una sustancial diferencia en el interior de cada familia (PÉREZ DE VARGAS \& Mártinez Calvo, 2000: 150). Sólo en el caso de las leguminosas situadas a menor altitud se muestra una asimetría más o menos marcada que podría hacernos pensar en desechar, en este único supuesto, la hipótesis de normalidad. Por otra parte, los outliers o datos de la muestra que parecen no ser coherentes con la mayoría de los registros (JoHnson, 1998: 25), y que nos señalan individuos que se encuentran por encima o por debajo de un rango y medio intercuartil (Сово \& al., 2008: 37), nos indicarían un posible error o accidente en el registro de datos. Siempre considerados con cierta cautela, y en vista de que las comparaciones entre escombreras situadas a diferentes altitudes y las totales no parecen mostrar diferencias significativas, se han confeccionado todos los cálculos incluyendo a dichos valores (Milton, 1994: 45), ya que los datos anómalos sólo deben rechazarse cuando existan fundadas sospechas de que no corresponden a un valor de la variable que estudiamos (García Nogales, 2004: 20).

\section{RESULTADOS}

El listado florístico confeccionado a partir de la prospección de dieciocho escombreras, localizadas en la zona centro-norte de la provincia de
Valladolid (España), aparece en el Anexo, y se eleva a 336 especies, incluidas en 209 géneros y 45 familias. Estos taxones, en forma de números absolutos y porcentajes de los grandes grupos clásicos de plantas vasculares, se reflejan en la Tabla 1. En ella destacan los nulos valores en el grupo de los pteridófitos y anecdóticos en el caso de las gimnospermas, tanto a nivel de familias como a nivel de especies (únicamente a través de la presencia de un pimpollo de Pinus halepensis localizado en el montículo de una escombrera). Las familias Liliaceae, y sobre todo Gramineae, son las únicas monocotiledóneas, dentro del gran grupo de Angiospermas, dominadas con más del $86 \%$ de las especies y del $93 \%$ de las familias por dicotiledóneas.

En la Tabla 2 aparecen las diez familias con mayor número de especies. Entre las cuatro primeras, Compositae, Gramineae, Leguminosae y Cruciferae, suman más del $50 \%$ del total de las especies y casi el $50 \%$ de los géneros. En ese grupo, no resulta demasiado sorprendente la presencia de familias como Chenopodiaceae y $\mathrm{Pa}$ paveraceae, dada la época del año (13 de junio de 2009 a 2 de julio de 2009) o el tipo de hábitat en que se realizó el estudio. Es destacable que las familias en que la relación $n^{\circ}$ géneros $/ n^{\circ}$ especies es mayor han sido Boraginaceae $(0,89)$, Umbelliferae $(0,79)$ y Caryophyllaceae $(0,73)$, coincidiendo las dos primeras familias con otros trabajos realizados en la provincia de Valladolid (LÁZARo BELLO, 2003, 2006, 2006b, 2007, 2008).

La comparación de los porcentajes de aparición de las principales familias en las escombreras, bien en función del número de especies, bien

Tabla 1

Distribución taxonómica de los grandes grupos de plantas vasculares.

\begin{tabular}{lcccc}
\hline \multirow{2}{*}{ GRUPO TAXONÓMICO } & \multicolumn{2}{c}{ FAMILIAS } & \multicolumn{2}{c}{ ESPECIES } \\
& Total & $\%$ & Total & 0,00 \\
\hline Pteridophyta s. $a$. & 0 & 0,00 & 0 & 0,30 \\
Pinophyta & 1 & 2,22 & 1 & 99,70 \\
Magnoliophyta & 44 & 97,78 & 335 & $(13,09)$ \\
$\quad($ Liliopsida) & $(2)$ & $(4,45)$ & $(44)$ & $(86,61)$ \\
$\quad$ Magnoliopsida) & $(42)$ & $(93,33)$ & $(291)$ & 100,00 \\
\hline TOTAL & 45 & 100,00 & 336 & \\
\hline
\end{tabular}


Tabla 2

Datos taxonómicos de los géneros y especies de las principales familias con representación en las zonas de estudio.

\begin{tabular}{|c|c|c|c|c|c|}
\hline \multirow{2}{*}{ FAMILIAS } & \multicolumn{2}{|c|}{ ESPECIES } & \multicolumn{2}{|c|}{ GÉNEROS } & \multirow{2}{*}{$\begin{array}{c}\text { Relación } \\
\mathrm{n}^{\circ} \text { géneros } / \mathrm{n}^{\circ} \text { especies }\end{array}$} \\
\hline & $\mathrm{n}^{\mathrm{o}}$ & $\%$ & $\mathrm{n}^{\mathrm{o}}$ & $\%$ & \\
\hline Compositae & 76 & 22,62 & 48 & 22,97 & 0,63 \\
\hline Gramineae & 42 & 12,50 & 27 & 12,92 & 0,64 \\
\hline Leguminosae & 30 & 8,93 & 14 & 6,70 & 0,47 \\
\hline Cruciferae & 21 & 6,25 & 14 & 6,70 & 0,67 \\
\hline Labiatae & 20 & 5,95 & 11 & 5,26 & 0,55 \\
\hline Umbelliferae & 14 & 4,17 & 11 & 5,26 & 0,79 \\
\hline Caryophyllaceae & 11 & 3,27 & 8 & 3,83 & 0,73 \\
\hline Chenopodiaceae & 10 & 2,98 & 5 & 2,39 & 0,50 \\
\hline Boraginaceae & 9 & 2,68 & 8 & 3,83 & 0,89 \\
\hline Papaveraceae & 8 & 2,38 & 3 & 1,43 & 0,38 \\
\hline TOTAL PARCIAL & 241 & 71,73 & 149 & 71,29 & \\
\hline Resto de familias & 95 & 28,27 & 60 & 28,71 & \\
\hline TOTAL & 336 & 100,00 & 209 & 100,00 & \\
\hline
\end{tabular}

en función del número de presencias, aparece recogida en la Figura 2, con gran paralelismo en los datos, pero sobresaliendo el ascenso, al considerar el número de presencias, en las familias Compositae, Cruciferae y Gramineae, y descenso, por contra, de la familia Leguminosae, aunque siempre con porcentajes inferiores al $5 \%$.

El desdoblamiento del total de las escombreras estudiada en dos grandes grupos, uno asentado sobre sustratos arcillosos y/o margosos, a altitudes de 760-820 m, y el otro sobre sustratos calcáreos (a veces margo-calcáreos), a altitudes de 840-850 $\mathrm{m}$, permite poner de manifiesto lo siguiente:

1.- Al nivel de confianza del $95 \%$, no hay diferencias significativas en el número medio de especies entre los dos grupos de escombreras $\left(F_{1,16}=0,7368, p\right.$-valor $=0,4034 ; B=1,0603, p$ valor $=0,3481$ ), si bien es superior en las escombreras asentadas en las parameras calizas $(x=86,88 \pm 27,27$ en las escombreras situadas a $760-820 \mathrm{~m} ; x=96,30 \pm 19,35$ en las situadas a 840-850 m). Las especies que han aparecido en todas las escombreras han sido Anacyclus clavatus, Eryngium campestre, Hordeum murinum, Lactuca serriola, Medicago sativa, Onopordum nervosum, Papaver rhoeas y Plantago lanceolata, y sólo han faltado en una escombrera las especies Bromus diandrus, Bromus madritensis, Carduus tenuiflorus y
Silybum marianum. Los géneros con mayor número de especies han sido Bromus y Centaurea, con 7 especies, y Carduus, Galium, Sisymbrium y Medicago, con 5 especies (después hay nueve géneros con cuatro especies cada uno, y catorce géneros con tres especies).

El número de especies presentes exclusivamente en escombreras arcilloso-margosas ha sido de $58(17,26 \%)$, en las escombreras calcáreas $93(27,68 \%)$, e indistintamente en unas u otras $185(55,06 \%)$.

2.- A simple vista, tampoco hay diferencias notables en el estudio de los biotipos y elementos corológicos (Tabla 3), lo que se ve confirmado por los análisis estadísticos (para biotipos, $F_{1,8}<0,0001, p$-valor $=1,0000 ; B=1,0100, p$ valor $=0,7901, \mathrm{y}$, para los elementos corológi$\cos , F_{1,10}<0,0001$, $p$-valor $=1,0000 ; B=1,0059$, $p$-valor $=0,8177)$.

Entre terófitos y hemicriptófitos se suma, tanto a nivel global de escombreras, como por grupos, valores porcentuales del $80 \%$ o más, siendo de alrededor del $50 \%$ el valor de los terófitos. La presencia de geófitos y fanerófitos es poco menos que anecdótica, y los valores de los caméfitos vienen marcados, básicamente, por especies de la familia Labiatae, de tanta importancia en páramos y cuestas vallisoletanos (LÁZARo BELLO, 2008: 17). 

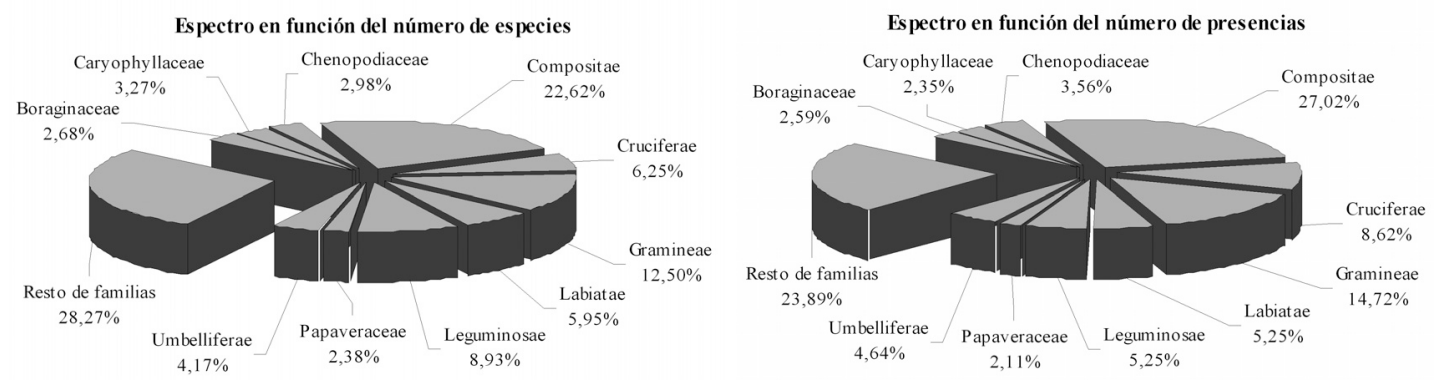

Figure 2. - Diagramas de sectores con representación de las principales familias botánicas.

El elemento corológico mediterráneo, en sentido amplio, es de alrededor del $50 \%$, aunque superior en zonas de páramos calcáreos, con porcentajes de endemicidad ibérica que no llega al $5 \%$,y, por tanto, acordes con los datos conocidos para las zonas centrales de la cuenca del Duero (Gómez-CAMPo \& Malato Beliz, 1985: 49; Sainz Ollero \& Moreno Saiz, 2002: 185), aunque no tanto si consideramos el tipo de sustrato estudiado o el biotipo hacia el que se focaliza la investigación, ya que trabajos como los de BuRgaz (1983: 125) o LÁ-
ZARO BELlo (2008: 17), aportan valores algo superiores. Por otra parte, a menor altitud, en zonas próximas a los fondos de valle, más transitadas y ocupadas por humanos, dominan los elementos de amplia distribución. A este respecto, conviene destacar el $6,25 \%$ de especies alóctonas (un total de 21 taxones, de los que un $66,67 \%$ de ellos se han localizado en las zona margo-arcillosas, y un $85,71 \%$ se han presentado en las calcáreas, aunque el análisis cuantitativo equilibra casi al cincuenta por ciento esos datos), valores algo superiores a los en-

Tabla 3

Resumen, en forma de porcentaje, de los espectros biológico y corológico. ( $\mathrm{n}=$ número de escombreras).

\begin{tabular}{|c|c|c|c|c|}
\hline TIPOS BIOLÓGICOS & & $\begin{array}{c}\text { Total } \\
\text { Escombreras } \\
(n=18)\end{array}$ & $\begin{array}{c}\text { Escombreras } \\
760-820 \mathrm{~m} \\
(\mathrm{n}=8)\end{array}$ & $\begin{array}{c}\text { Escombreras } \\
840-850 \mathrm{~m} \\
(\mathrm{n}=10)\end{array}$ \\
\hline Terófitos & & 50,89 & 54,32 & 47,48 \\
\hline Geófitos & & 2,68 & 2,88 & 3,24 \\
\hline Hemicriptófitos & & 30,95 & 31,69 & 32,38 \\
\hline Caméfitos & & 12,80 & 9,05 & 15,10 \\
\hline \multirow[t]{2}{*}{ Fanerófitos } & & 2,68 & 2,06 & 1,80 \\
\hline & TOTAL & 100,00 & 100,00 & 100,00 \\
\hline ELEMENTOS COROLÓGICOS & & $\begin{array}{c}\text { Total } \\
\text { Escombreras } \\
(n=18)\end{array}$ & $\begin{array}{c}\text { Escombreras } \\
760-820 \mathrm{~m} \\
(\mathrm{n}=8)\end{array}$ & $\begin{array}{c}\text { Escombreras } \\
840-850 \mathrm{~m} \\
(\mathrm{n}=10)\end{array}$ \\
\hline Endemismo ibérico & & 3,57 & 2,47 & 3,96 \\
\hline Ibero-norteafricano & & 1,19 & 1,23 & 1,08 \\
\hline Mediterráneo occidental & & 14,88 & 12,76 & 15,83 \\
\hline Mediterráneo & & 32,44 & 30,45 & 30,57 \\
\hline Elemento mediterráneo total & & 52,08 & 46,91 & 51,44 \\
\hline Elemento eurosiberiano & & 5,36 & 5,35 & 5,76 \\
\hline \multirow[t]{2}{*}{ Elemento de amplia distribución } & & 42,56 & 47,74 & 42,80 \\
\hline & TOTAL & 100,00 & 100,00 & 100,00 \\
\hline
\end{tabular}


Tabla 4

Porcentaje medio de especies en las principales familias presentes en las escombreras estudiadas. Los datos se refieren al valor medio y a la desviación estándar y, entre paréntesis, se dan los valores mínimo y máximo. ( $n=$ número de escombreras).

\begin{tabular}{lccc}
\hline FAMILIAS & $\begin{array}{c}\text { Total } \\
\text { Escombreras } \\
(\mathrm{n}=18)\end{array}$ & $\begin{array}{c}\text { Escombreras } \\
760-820 \mathrm{~m} \\
(\mathrm{n}=8)\end{array}$ & $\begin{array}{c}\text { Escombreras } \\
840-850 \mathrm{~m} \\
(\mathrm{n}=10)\end{array}$ \\
\hline Compositae & $26,89 \pm 3,77$ & $27,34 \pm 4,62$ & $26,53 \pm 3,15$ \\
& $(20,89-34,78)$ & $(22,06-34,78)$ & $(20,89-32,11)$ \\
Cruciferae & $8,70 \pm 2,29$ & $8,33 \pm 2,39$ & $9,00 \pm 2,30$ \\
& $(4,92-13,43)$ & $(4,92-11,43)$ & $(5,51-13,43)$ \\
Gramineae & $14,92 \pm 2,57$ & $15,35 \pm 2,27$ & $14,57 \pm 2,86$ \\
& $(10,67-18,58)$ & $(12,07-18,03)$ & $(10,67-18,58)$ \\
Leguminosae & $4,83 \pm 2,73$ & $5,23 \pm 3,64$ & $4,50 \pm 1,86$ \\
& $(1,33-12,59)$ & $(2,67-12,59)$ & $(1,33-6,85)$ \\
\hline
\end{tabular}

contrados en otros estudios provinciales, que abarcaban el mismo tipo de sustratos, pero no tan alterados (BURGAZ, 1983; ROMERO MARTíN \& RICO, 1989; LÁZARO BELLO, 2006b; 2007).

3.- La suma de las especies de las cuatro principales familias siempre es superior al $50 \%$. Si las escombreras se dividen en grupos (Tabla 4) nunca hay diferencias significativas en los valores porcentuales específicos de dichas familias (para Compositae, $F_{1,16}=0,1951, p$ valor $=0,6646 ; B=1,0753$, $p$-valor $=0,2961$; para Cruciferae, $F_{1,16}=0,3707, \quad p$-valor $=0,5512$; $B=1,0008, p$-valor $=0,9155$; para Gramineae, $F_{1, l 6}=0,4042, p$-valor $=0,5339 ; B=1,0262, p$ valor $=0,5327$; para Leguminosae, $F_{1,16}=0,3077$, $p$-valor $=0,5868 ; B=1,2449, p$-valor $=0,0695)$, aunque, en función del tipo de escombreras, sí se ponen de manifiesto algunas variaciones, a veces notables, en los espectros biológicos y corológicos de dichas familias (Tabla 5). Así, mientras la mayoría de las especies de la familia Compositae se reparten, de forma no muy diferente, entre terófitos y hemicriptófitos, en el resto de familias predominan los terófitos, con el caso más notable de Cruciferae, en donde más del $80 \%$ de sus especies presentan este tipo biológico.

Del espectro corológico es preciso destacar el dato de más del $55 \%$ de elementos mediterráneos en Compositae y Leguminosae. Los endemismos ibéricos corresponden a es- casos taxones: Carduus assoi subsp. assoi, Carduus platypus subsp. granatensis y Onopordum nervosum, para la familia Compositae, Diplotaxis virgata y Erysimum mediohispanicum, para la familia Cruciferae, e Hippocrepis commutata, para la familia Leguminosae, no habiendo aparecido endemismos ibéricos de la familia Gramineae. Abundando en todo ello, los diagramas de cajas y patillas de la Figura 3 nos ofrecen información visual sobre la distribución de datos en estas cuatro familias.

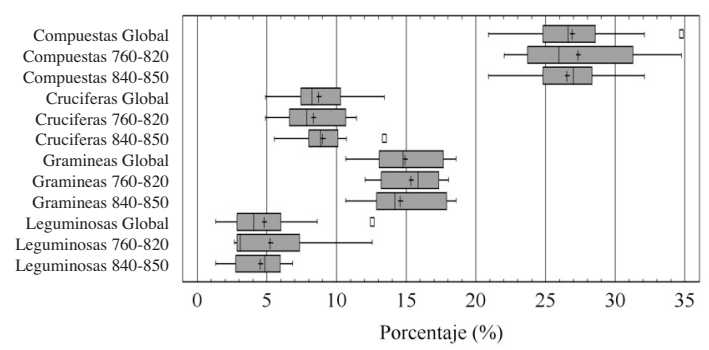

Figura 3. - Diagrama de caja y patillas para las cuatro principales familias botánicas representadas en las escombreras estudiadas.

\section{DISCUSIÓN}

El análisis conjunto de todos los datos nos permite destacar la importancia de terófitos y hemicriptófitos como biotipos más frecuentes en las escombreras prospectadas en la época de estudio, 
Tabla 5

Resumen global, en forma de porcentaje, de los espectros biológico y corológico en las principales familias encontradas en los medios estudiados.

\begin{tabular}{|c|c|c|c|c|c|}
\hline $\begin{array}{l}\text { TIPOS BIOLÓGICOS } \\
\text { (datos globales) }\end{array}$ & & Compositae & Cruciferae & Gramineae & Leguminosae \\
\hline Terófitos & & 40,79 & 80,95 & 64,29 & 63,34 \\
\hline Geófitos & & 1,32 & 0,00 & 7,14 & 0,00 \\
\hline Hemicriptófitos & & 48,68 & 19,05 & 28,57 & 20,00 \\
\hline Caméfitos & & 9,21 & 0,00 & 0,00 & 13,33 \\
\hline \multirow[t]{2}{*}{ Fanerófitos } & & 0,00 & 0,00 & 0,00 & 3,33 \\
\hline & TOTAL & 100,00 & 100,00 & 100,00 & 100,00 \\
\hline $\begin{array}{l}\text { ELEMENTOS COROLÓGICOS } \\
\text { (datos globales) }\end{array}$ & & Compositae & Cruciferae & Gramineae & Leguminosae \\
\hline Endemismo ibérico & & 3,95 & 9,52 & 0,00 & 3,33 \\
\hline Ibero-norteafricano & & 0,00 & 0,00 & 0,00 & 6,67 \\
\hline Mediterráneo occidental & & 17,10 & 19,05 & 11,91 & 16,67 \\
\hline Mediterráneo & & 35,53 & 14,29 & 33,33 & 33,33 \\
\hline Elemento mediterráneo total & & 56,58 & 42,86 & 45,24 & 60,00 \\
\hline Elemento eurosiberiano & & 11,84 & 0,00 & 9,52 & 0,00 \\
\hline Elemento de amplia distribución & & 31,58 & 57,14 & 45,24 & 40,00 \\
\hline & TOTAL & 100,00 & 100,00 & 100,00 & 100,00 \\
\hline
\end{tabular}

así como el ligero predominio del elemento corológico mediterráneo sobre el de amplia distribución (Figura 4), y unos porcentajes de endemicidad ibérica que no desentonan con respecto a lo sabido gracias a otros trabajos publicados realizados en zonas próximas de la provincia de Valladolid (LÁZARO BELLO, 2006b; 2007; 2009).

También trabajos como el actual permiten poner de manifiesto especies de interés provincial, bien por ser novedades provinciales (Ammi majus), bien por haber sido muy poco citadas (As-

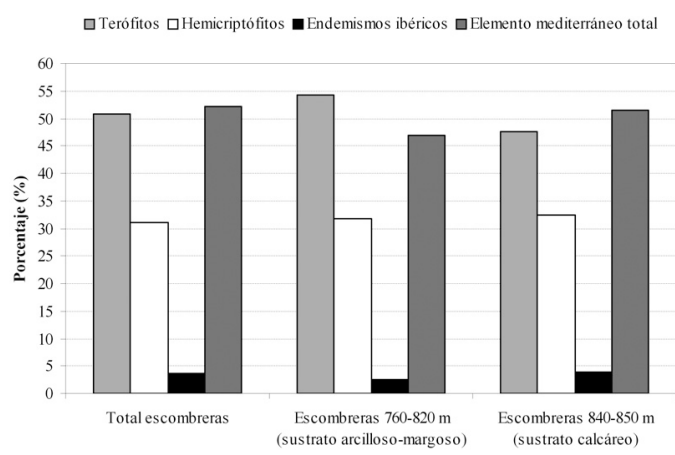

Figura 4. - Diagrama de barras resumen con los más destacados biotipos y comparación de la endemicidad ibérica con la mediterránea total. tragalus cymbaecarpos, Ecballium elaterium subsp. elaterium, Lappula squarrosa, etc.), bien por ser alóctonas, algunas también escasamente citadas (Consolida ajacis, Lonicera japonica, Phalaris canariensis, etc.), bien por conocerse en la provincia, pero no haber sido mencionadas como asilvestradas (Lens culinaris, Solanum tuberosum, Zea mays), bien por la presencia de endemismos ibéricos, algunos típicos ruderales (como los cardos citados anteriormente) y otros procedentes de las áreas adyacentes y aparentemente fuera de lugar (Dianthus pungens subsp. hispanicus, Sideritis spinulosa, Thymus mastigophorus, etc.). Sorprende algo la presencia de algunos fanerófitos (Quercus ilex subsp. ballota, Pinus halepensis, Populus nigra, etc.), aunque, normalmente, se trataba de pimpollos o individuos poco desarrollados, también provinientes de zonas cercanas. No hay que olvidar que el recubrimiento de los montículos de escombros por el sustrato típico de la región permite la recuperación progresiva de la vegetación potencial del territorio (JunTA DE CASTILLA Y LEÓN, 1997: 18), y eso es lo que se pone de manifiesto al comprobar el listado florístico de algunas de las escombreras. 
La época en que se ha realizado la investigación (finales de primavera a comienzos de verano) condiciona la ausencia de muchas especies de terófitos que tuvieron su apogeo en semanas anteriores (Medicago spp., Trifolium spp., etc.), $\mathrm{y}$, al contrario facilita la aparición de otras más tardías (Amaranthus spp., Chenopodium spp., etc.). En este sentido, será interesante realizar futuros trabajos dirigidos a averiguar las diferencias fenológicas que se producen en los distintos tipos de escombreras, así como el posible efecto ejercido por el sustrato, con un análisis más pormenorizado sobre la naturaleza del material vertido y su influencia sobre la flora.

\section{BIBLIOGRAFÍA}

Aizpuru, I., Aseginolaza, C., Uribe-Echebarría, P.M., Urrutia, P. \& Zorrakín, I. (eds.) -1999- Claves ilustradas del País Vasco y Territorios limítrofes - Servicio Central de Publicaciones del Gobierno Vasco. Vitoria-Gasteiz. $831 \mathrm{pp}$.

Bolòs, O. \& Vigo, J. - 1984-2001 - Flora dels Països Catalans. Vols. I-IV - Editorial Barcino. Barcelona

Burgaz, A.R. - 1983 - Flora y vegetación gipsófila de la provincia de Valladolid y sureste de la de Palencia Diputación Provincial de Valladolid e Institución Cultural Simancas. Valladolid. 200 pp.

Castroviejo, S. \& al. (coords.) - 1986-2010 - Flora iberica. Plantas vasculares de la Península Ibérica e Islas Baleares - Real Jardín Botánico, C.S.I.C. Madrid.

Cobo, E., Muñoz, P. \& González, J.A. -2008 - Bioestadística para no estadísticos. Bases para interpretar artículos científicos - Elsevier Masson. Barcelona. 354 pp.

Devesa Alcaraz, J.A. - 1995- Vegetación y flora de Extremadura - Universitas Editorial. Badajoz. 773 pp.

Diputación de Valladolid -2010 - Accesible en Internet en http://www.diputacióndevalladolid.es/medioambiente, en http://www.diputacióndevalladolid.es/prensa, en http:// www.derecho.com/l/bop-valladolid y en http://www.actualidad24horas.com. Consulta realizada en marzo de 2010.

Font Quer, P. - 1983 - Diccionario de Botánica - Ediciones Península. Barcelona. 1244 pp.

García Fernández, J. - 1986- El clima en Castilla y León - Ediciones Ámbito. Valladolid. 370 pp.

García Nogales, A. -2004- Bioestadística básica - Universidad de Extremadura. Badajoz. 266 pp.

Gómez Campo, C. \& Malato-Beliz, J. - 1985 - The Iberian Peninsula - In: Gómez-Campo, C. (Ed.). Plant Conservation in the Mediterranean Area, 47-70 DR W Junk. La Haya. 236 pp.

Hernández López, J.M. -1997 - Frecuentación por aves de los vertederos de Ávila - Medio Ambiente en Castilla y León 8: 17-22.

Hernández Pacheco, E. -1915- Geología y Paleontología del Mioceno de Palencia - Junta Ampl. Est. e Inv. Cientif. Comunicación de Inv. Paleont. y Prehist. 5: 1-75.

Johnson D.E. - 1998 - Métodos multivariados aplicados al análisis de datos - International Thomson Editores. México. 566 pp.
Junta de Castilla y León - 1997- Recuperación de suelo contaminado en Boecillo, Valladolid - Conserjería de Medio Ambiente y Ordenación del Territorio, Informe Técnico. 46 pp.

Junta de Castilla y León - 2000 - Dossier: objetivos de la estrategia de residuos para Castilla y León - Calidad Ambiental 16: 4-5.

Junta de Castilla y León - 2001 - Dossier: directiva de vertederos - Calidad Ambiental 17: 4-5.

Ladero Álvarez, M., Navarro Andrés, F. \& Valle Gutiérrez, C.J. - 1983- Comunidades nitrófilas salmantinas Stud. Bot. 2: 7-67.

Ladero Álvarez, M. Valle Gutiérrez, C.J., Santos Bobillo, M.T., Fernández Arias, M.I. \& Amor Morales, A. 1987-88 - Aproximación hacia una síntesis de las comunidades nitrófilas del CW español y su relación con las series de vegetación - Lazaroa 10: 11-22.

Lázaro Bello, J.A. - 2003 - Algunas plantas interesantes de los Montes de Torozos (Valladolid, España) - Lazaroa 24: 127-128.

Lázaro Bello, J.A. - 2005 - Estudio de la diversidad florística en páramos de la zona centro de la cuenca del Duero (Valladolid, España) - Toll Negre 6: 2534.

Lázaro Bello, J.A. -2006- Más plantas interesantes de los Montes de Torozos (Valladolid, España) - Lazaroa 27: 137-140.

Lázaro Bello, J.A. - 2006a - Estudio de la diversidad florística en cuestas de la zona centro de la cuenca del Duero (Valladolid, España) - Toll Negre 7: 19-30.

Lázaro Bello, J.A. - 2006b - Renedo de Esgueva (Valladolid, España): Catálogo florístico y análisis de resultados - Ecología 20: 163-216.

Lázaro Bello, J.A. -2007- Análisis biogeográfico de la flora vascular del tramo bajo del valle del Esgueva (Valladolid, España) - Toll Negre 9: 23-32.

Lázaro Bello, J.A. - 2008 - Algunas gramíneas de interés corológico en la provincia de Valladolid (España) - Lazaroa 29: 139-142.

Lázaro Bello, J.A. - 2008- Estudio sobre la diversidad de caméfitos en el centro de la cuenca del Duero (España) - Lagascalia 28: 7-20.

Lázaro Bello, J.A. -2009a - Caracterización de comunidades vegetales de Hyoscyamus niger L. (Solanaceae) 
en la provincia de Valladolid (España) - Anales de Biología, Univ. Murcia 31: 121-133.

Lázaro Bello, J.A. - 2009c - Nueva contribución al conocimiento de Gramineae Juss. en la provincia de Valladolid (España) - Toll Negre 11: 97-102.

Mateo Sanz, G. \& Crespo, M.B. -2009- Manual para la determinación de la flora valenciana - Librería Compas. Alicante. 507 pp.

Milton, J. - 1994- Estadística para Biología y Ciencias de la Salud - McGraw-Hill-Interamerica de España. Madrid. 519 pp.

Penas Merino, A., Díaz González, T.E., García González, M.E., López Pacheco, M.J., Puente García, E. \& Herrero Cembranos, L. - 1987-88 - Datos sobre los cardales y tobales (Onopordetea acanthii) en la provincia de León - Lazaroa 10: 65-79.

Pérez de Vargas, A. \& Martínez Calvo, M.C. -2000 - Estadística Biométrica - Editorial Síntesis. Madrid. 221 pp.

Rivas-Martínez, S., Díaz, T.E., Fernández-González, F., Izco, J., Loidi, J., Lousa, M. \& Penas, A. -2002 - Vascular Plant Communities of Spain and Portugal. Addenda to the Syntaxonomical Checklist of 2001 Itinera Geobotanica 15(1): 5-432.
Romero Martín, T. \& Rico, E. - 1989- Flora de la cuenca del río Duratón - Ruizia 8: 1-438.

Sainz Ollero, H. \& Moreno Saiz, J.C. - 2002 - Flora vascular. In Pineda, F.D. \& al. (eds.): La diversidad biológica de España, 175-195 - Prentice Hall. Madrid. 412 pp.

Sánchez Sánchez, J. \& Fernández Díez, F.J. - 1988 Notas sobre flora vallisoletana - Lazaroa 9: 181-187.

Triska, J. - 1987 - Flore d'Europe - Gründ. Paris. 298 pp.

Tutin, T.G., Burges, N.A., Edmonson, J.R., Heywood, V.H., Moore, D.M., Valentine, D.H., Walters, S.M. \& Webb, D.A. (eds.) 1993 - Flora europaea. Volume 1. Psilotaceae to Platanaceae - Cambridge University Press. Great Britain. 581 pp.

Tutin, T.G., Heywood, V.H., Burges, N.A., Valentine, D.H., Walters, S.M. \& Webb, D.A. (eds.) -1964-1980Flora europaea. 5 vols. [en los vols. 2-5, a los editores mencionados se suma Moore, D.M.] - Cambridge University Press. Great Britain.

Villar, L., Sesé, J.A. \& Ferrández, J.V. -1997-2001 - Flora del Pirineo Aragonés. Vols. I-II. Instituto de Estudios Altoaragoneses y Consejo de Protección de la Naturaleza de Aragón. Huesca. 


\begin{abstract}
ANEXO 1
A continuación se presenta, por orden de familia, género y especie, el listado de taxones inventariados en el presente trabajo. Se incluye el nombre científico con la autoría correspondiente, el biotipo y la corología. Para los tipos biológicos y para los elementos corológicos las abreviaturas adoptadas han sido: F-fanerófito, C-caméfito, H-hemicriptófito, G-geófito, T-terófito, Neof-neófito, Subcosmsubcosmopolita, Plur-plurirregional, Eurosib-eurosiberiano, Med-mediterráneo, Med W-mediterráneo occidental, Ib-nort-iberonorteafricano, End-ib-endemismo ibérico. Los números son indicativos de las correspondientes escombreras (véase el apartado Material y Métodos).
\end{abstract}

\title{
Amaranthaceae
}

Amaranthus albus L., T, Neof, 5, 6, 8, 12, 17.

Amaranthus deflexus L., C, Neof, 3, 15, 16, 18.

Amaranthus retroflexus L., T, Neof, 3, 6, 12, 14, 15, 16, 17.

\section{Boraginaceae}

Anchusa azurea Mill., H, Med, 1, 3, 8, 17.

Asperugo procumbens L., T, Plur, 2, 3, 5, 6, 7, 8, 9, 12, 18.

Buglossoides arvensis (L.) I.M. Johnston, T, Plur, 5, 7, 8.

Cynoglossum cheirifolium L., H, Med W, 3 .

Echium asperrimum Lam., H, Med W, 1, 2, 3, 8, 10, 13, 14, 15, 18.

Echium vulgare L., H, Eurosib, 1, 2, 3, 4, 8, 9, 11, 12, 13, 14, 15, 17, 18.

Lappula squarrosa (Retz.) Dumort., T, Eurosib, 11.

Lithodora fruticosa (L.) Griseb., C, Med W, 4, 11.

Neatostema apulum (L.) I.M. Johnston, T, Med, 11.

\section{Caprifoliaceae}

Lonicera japonica Thunb., F, Neof, 1.

\section{Caryophyllaceae}

Arenaria serpyllifolia L., T, Subcosm, 1.

Dianthus pungens subsp. hispanicus (Asso) O. Bolòs \& Vigo, C, End-ib, 4.

Herniaria cinerea DC., T, Med, 1, 3, 8, 9, 12, 13, 14, 17, 18.

Paronychia capitata (L.) Lam. subsp. capitata, H, Med, 14.

Petrorhagia nanteuilii (Burnat) P.W. Ball \& Heywood, T, Med W, 1, 4, 8, 9, 10, 11, 14.

Scleranthus delortii Gren., T, Med W, 1.

Silene conica L. subsp. conica, T, Med, 4.

Silene legionensis Lag., H, Med W, 10.

Silene nocturna L., T, Subcosm, 4, 14, 15.

Silene vulgaris (Moench) Garcke subsp. vulgaris, H, Subcosm, 1, 2, 4, 5, 6, 7, 8, 9, 10, 14, 15, 18.

Stellaria pallida (Dumort.) Piré, T, Plur, 5, 9.

\section{Chenopodiaceae}

Atriplex patula L., T, Plur, 8, 9, 12, 13.

Atriplex prostrata Boucher ex DC., T, Plur, 11, 16, 17.

Atriplex rosea L., T, Plur, 1, 3, 5, 6, 7, 9, 12, 13, 16, 18.

Bassia prostrata (L.) Beck, C, Plur, 4, 13, 16.

Beta vulgaris L., H, Plur, 1.

Chenopodium album L., T, Subcosm, 1, 2, 5, 6, 8, 9, 12, 13, 14, 15, 16, 17, 18.

Chenopodium multifidum L., H, Neof, 5, 17. 
Chenopodium opulifolium Schrad. ex Koch \& Ziz, T, Subcosm, 3, 5, 6, 7, 8, 9, 11, 12, 16.

Chenopodium vulvaria L., T, Subcosm, 5, 7, 9, 12, 15, 16.

Salsola kali L., T, Plur, 3, 5, 6, 7, 8, 11, 12, 17.

\section{Cistaceae}

Helianthemum cinereum subsp. rotundifolium (Dunal) Greuter \& Burdet, C, Med W, 4, 9, 10, 11 .

Helianthemum hirtum L., C, Med W, 1, 4, 10, 11, 14, 15, 16.

Helianthemum ledifolium (L.) Mill., T, Med, 8, 10.

Helianthemum salicifolium (L.) Mill., T, Med, 9, 14.

\section{Compositae}

Achillea millefolium L. subsp. millefolium, $\mathrm{H}$, Eurosib, 6 .

Achillea odorata L., H, Med W, 4, 6, 8, 9, 10, 11, 14, 18.

Anacyclus clavatus (Desf.) Pers., T, Med, 1, 2, 3, 4, 5, 6, 7, 8, 9, 10, 11, 12, 13, 14, 15, 16, 17, 18.

Andryala integrifolia L., H, Med, 8, 12, 13, 14, 15, 17.

Andryala ragusina $\mathrm{L} ., \mathrm{H}$, Med W, 7, 14.

Anthemis cotula L., T, Plur, 7, 12, 13 .

Arctium minus (Hill.) Bernh., H, Eurosib, 8.

Artemisia absinthium L., C, Eurosib, 6, 7 .

Artemisia campestris subsp. glutinosa (J. Gay ex Besser) Batt., C, Med W, 1, 10, 11, 15, 18.

Artemisia herba-alba Asso, C, Plur, 10, 13, 16.

Asteriscus aquaticus (L.) Less., T, Med, 13.

Bombycilaena erecta (L.) Smolj., T, Med, 1, 10, 11.

Calendula officinalis L., T, Neof, 6.

Carduncellus monspelliensium All., H, Med, 10, 11.

Carduus assoi (Willk.) Devesa \& Talavera subsp. assoi, H, End-ib, 8, 14.

Carduus bourgeanus Boiss. \& Reut. subsp. bourgeanus, T, Med W, 1, 3, 4, 5, 6, 7, 9, 11, 12, 13.

Carduus platypus subsp. granatensis (Willk.) Nyman, T, End-ib, 1, 4, 8, 9, 10.

Carduus pycnocephalus L., T, Med, 1, 2, 3, 4, 8, 9, 11, 13, 15, 17.

Carduus tenuiflorus Curtis, T, Med, 2, 3, 4, 5, 6, 7, 8, 9, 10, 11, 12, 13, 14, 15, 16, 17, 18.

Carlina corymbosa $\mathrm{L}$. subsp. corymbosa, $\mathrm{H}, \mathrm{Med}, 4,6,9,10,11,16,18$.

Carthamus lanatus L. subsp. lanatus, T, Med, 1, 3, 8, 11, 13, 14, 16, 18.

Centaurea alba L., C, Med, 1.

Centaurea aspera L., C, Med W, 2, 3, 4, 5, 6, 7, 8, 9, 10, 11, 14, 15, 16, 18.

Centaurea calcitrapa L., H, Med, 1, 3, 5, 7, 8, 9, 10, 12, 14, 16, 18.

Centaurea melitensis L., T, Med W, 1, 5, 9, 11, 13, 14, 17, 18.

Centaurea ornata Willk. subsp. ornata, H, Med W, 4, 8, 10, 11, 15.

Centaurea scabiosa L., H, Eurosib, 2, 6, 8, 9, 10, 14.

Centaurea solstitialis L. subsp. solstitialis, T, Plur, 8 .

Chamomilla recutita (L.) Rauschert, T, Eurosib, 1.

Cichorium intybus L., H, Plur, 1, 2, 3, 5, 6, 7, 8, 10, 11, 12, 13, 14, 15, 16, 17, 18.

Cirsium arvense (L.) Scop., G, Plur, 1, 3, 5, 6, 11, 12, 13, 14.

Cirsium vulgare (Savi) Ten., H, Plur 1, 3, 10, 14.

Cnicus benedictus L., T, Med, 7.

Conyza canadensis (L.) Cronq., T, Neof, 1, 3, 12, 17.

Crepis capillaris (L.) Wallr., T, Plur, 1, 3, 4, 5, 6, 7, 8, 9, 10, 11, 14, 16.

Crepis pulchra L., T, Plur, 1, 2, 3, 4, 5, 6, 7, 9, 11, 12, 13.

Crepis vesicaria subsp. taraxacifolia (Thuill.) Thell. ex Schinz \& R. Keller, H, Eurosib, 4, 9, 13, 15, 16.

Crupina vulgaris Cass., T, Plur, 11, 14.

Evax carpetana Lange, T, Med W, 1. 
Filago pyramidata L., T, Plur, 1, 4, 8, 9, 10, 11, 14, 18.

Hedypnois cretica (L.) Dum.-Courset, T, Med, 13.

Helichrysum stoechas (L.) Moench subsp. stoechas, C, Med, 4, 11.

Hypochoeris radicata L., H, Plur, 12.

Inula montana $\mathrm{L} ., \mathrm{H}, \mathrm{Med} \mathrm{W}, 4,10,11,14$.

Lactuca saligna $\mathrm{L} ., \mathrm{T}$, Plur, 1 .

Lactuca serriola L., T, Plur, 1, 2, 3, 4, 5, 6, 7, 8, 9, 10, 11, 12, 13, 14, 15, 16, 17, 18.

Lactuca viminea (L.) J. \& C. Presl, H, Med, 9.

Leontodon taraxacoides (Vill.) Mérat, H, Eurosib, 1, 4, 10, 14, 18.

Leucanthemum pallens (Gay) DC., H, Med, 6, 11 .

Leuzea conifera (L.) DC., H, Med W, 10, 11.

Mantisalca salmantica (L.) Briq. \& Cavill., H, Med, 1, 3, 4, 5, 6, 7, 8, 9, 10, 11, 12, 13, 14, 15.

Onopordum acanthium L., H, Eurosib, 1, 2, 3, 4, 5, 6, 8, 9, 10, 11, 14, 15, 16, $17,18$.

Onopordum nervosum Boiss., H, End-ib, 1, 2, 3, 4, 5, 6, 7, 8, 9, 10, 11, 12, 13, 14, 15, 16, 17, 18.

Pallenis spinosa (L.) Cass. subsp. spinosa, $\mathrm{H}, \mathrm{Med}, 11$.

Picnomon acarna (L.) Cass., T, Med, 1, 4, 5, 7, 8, 10, 11, 14, 16.

Picris echioides L., T, Med, 1, 3, 11, 15, 18.

Picris hieracioides L., subsp. hieracioides, H, Plur, 1, 4, 5, 8, 11, 12, 17.

Pilosella pseudopilosella (Ten.) Sojàk, H, Med, 1, 6, 9, 18.

Santolina chamaecyparissus subsp. squarrosa (DC.) Nyman, C, Med W, 4, 9, 10, 11, $14,16$.

Scolymus hispanicus L., H, Med, 3, 5, 8, 13, 14, 15, 16, 18.

Scorzonera hirsuta L., H, Med W, 4.

Scorzonera laciniata L., H, Plur, 1, 4, 5, 6, 8, 10, 13, 14, 17.

Senecio gallicus Chaix, T, Med, 7.

Senecio jacobea L., H, Eurosib, 1, 2, 3, 4, 6, 7, 8, 11, 13.

Senecio vulgaris L., T, Subcosm, 5.

Serratula pinnatifida (Cav.) Poir., H, Med W, 11.

Silybum marianum (L.) Gaertn., H, Med, 1, 2, 3, 4, 5, 6, 7, 8, 9, 10, 12, 13, 14, 15, 16, 17, 18.

Sonchus asper (L.) Hill. T, Plur, 4, 7, 8, 9, 12, 13, 14, 15, 18.

Sonchus oleraceus L., T, Plur, 1, 2, 3, 5, 6, 7, 8, 9, 12, 14, 15, 16, 17.

Taraxacum gr. erythrospermum Andrz. ex Besser, H, Plur, 9, 12.

Taraxacum gr. officinale Weber, H, Plur, 3.

Tragopogon crocifolius L. subsp. crocifolius, H, Med, 3, 6, 13, 14.

Tragopogon dubius Scop., H, Plur, 2, 4, 8, 10, 11.

Tragopogon porrifolius L., H, Med, 5, 7, 8, 9, 14, 15, 17.

Xanthium spinosum L., T, Neof, 3, 5, 6, 12, 16.

Xeranthemum inapertum (L.) Mill., T, Med, 1, 4, 8, 10, 14.

\section{Convolvulaceae}

Convolvulus arvensis L., H, Subcosm, 1, 2, 3, 5, 6, 7, 8, 9, 11, 12, 13, 15, 17, 18.

Convolvulus lineatus L., H, Med, 1.

Cuscuta approximata Bab., T, Plur, 8, 10.

\section{Crassulaceae}

Pistorinia hispanica (L.) DC., T, End-ib, 1.

Sedum album L., C, Med, 8, 14, 15.

\section{Cruciferae}

Alyssum granatense Boiss. \& Reut., T, Med W, 9, 15.

Alyssum simplex Rudolphi, T, Plur, , 3, 4, 10. 
Biscutella auriculata $\mathrm{L} ., \mathrm{T}$, Med W, 1, 2, 3, 4, 6, 7, 8, 9, 10, 11.

Capsella bursa-pastoris (L.) Medik., T, Subcosm, 1, 2, 3, 5, 6, 7, 8, 10, 13, 14.

Cardaria draba (L.) Desv. subsp. draba, H, Plur, 1, 5, 6, 7, 8, 12, 14, 18.

Coronopus squamatus (Forssk.) Asch., T, Subcosm, 10.

Descurainia sophia (L.) Webb ex Prantl, T, Subcosm, 2, 3, 5, 6, 7, 8, 9, 10, 14, 18.

Diplotaxis erucoides (L.) DC. subsp. erucoides, T, Med, 1, 17.

Diplotaxis virgata (Cav.) DC. subsp. virgata, T, End-ib, 5, 6, 8, 13.

Eruca vesicaria (L.) Cav., T, Med, 1, 2, 3, 4, 5, 6, 8, 9, 10, 11, 12, 14, 15, 17, 18.

Erysimum mediohispanicum Polatschek, H, End-ib, 10.

Erysimum repandum L., T, Plur, 4.

Hirschfeldia incana (L.) Lagr.-Foss., H, Plur, 1, 2, 3, 4, 5, 6, 7, 8, 9, 10, 12, 14, $15,17$.

Neslia paniculata subsp. thracica (Velen.) Bornm., T, Plur, 2.

Rapistrum rugosum (L.) All. subsp. rugosum, T, Med, 1, 3, 4, 6, 8, 9, 10, 11, 12, 13, 16, 17, 18.

Sinapis arvensis L., T, Subcosm, 1, 2, 3, 4, 5, 6, 7, 8, 11, 12, 14, 15, 16, 17, 18.

Sisymbrium austriacum subsp. contortum (Cav.) Rouy \& Foucaud, H, Med W, 2, 4, 11.

Sisymbrium crassifolium Cav., T, Med W, 2, 3, 4, 7, 8, 9, 10, 11, 15, 17.

Sisymbrium irio L., T, Plur, 1, 3, 4, 5, 7, 8, 9, 10, 12.

Sisymbrium orientale L., T, Plur, 3, 5, 8, 14, 15, 16, 17, 18.

Sisymbrium runcinatum Lag. ex DC., T, Plur, 1, 9, 13.

\section{Cucurbitaceae}

Bryonia dioica Jacq., G, Med, 5, 7, 8 .

Ecballium elaterium (L.) A. Rich. subsp. elaterium, G, Plur, 1, 11.

\section{Dipsacaceae}

Cephalaria leucantha (L.) Roem. \& Schult., C, Med, 4.

Dipsacus fullonum L., H, Plur, 8, 9.

Lomelosia simplex (Desf.) Raf. subsp. simplex, T, Med W, 11.

Scabiosa atropurpurea L., H, Med, 1, 2, 3, 4, 5, 6, 8, 9, 10, 11, 12, 14, 15, 17, 18.

\section{Elaeagnaceae}

Elaeagnus angustifolia L., F, Neof, 5.

\section{Euphorbiaceae}

Euphorbia falcata L. subsp. falcata, T, Plur, 1.

Euphorbia serrata L., C, Med W, 1, 2, 4, 5, 6, 7, 8, 9, 10, 11, 13, 18.

Mercurialis tomentosa L., C, Med W, 11.

\section{Fagaceae}

Quercus ilex subsp. ballota (Desf.) Samp., F, Med, 4.

\section{Geraniaceae}

Erodium ciconium (L.) L'Hér., T. Med, 1, 3, 6, 8, 9, 12, 14, 15, 17, 18.

Erodium cicutarium (L.) L'Hér. subsp. cicutarium, T, Subcosm, 1, 3, 5, 10, 11, 14, 15, 16.

Erodium malacoides (L.) L'Hér., T, Med, 3, 5, 14.

Geranium dissectum L., T, Eurosib, 9, 10, 12, 13, 14, 17.

Geranium molle L., T, Plur, 1, 3, 9, 10, 14. 


\section{Gramineae}

Aegilops geniculata Roth., T, Med, 1, 4, 10, 11, 14.

Avena barbata Pott ex Link subsp. barbata, T, Plur, 4, 5, 10, 11, 12, 13, 14, 15, 16, 17, 18.

Avena sativa L., T, Plur, 5, 8, 14.

Avena sterilis subsp. ludoviciana (Durieu) Nyman, T, Med, 1, 2, 3, 6, 7, 9, 10.

Avenula bromoides subsp. pauneroi Romero Zarco, H, Med W, 1, 4, 9, 10, 11, 14.

Brachypodion distachyon (L.) Beauv., T, Med, 4, 10, 11.

Brachypodion phoenicoides (L.) Roem. \& Schult., H, Med W, 11, 14, 18.

Bromus diandrus Roth, T, Med, 1, 2, 3, 4, 5, 6, 7, 8, 9, 11, 12, 13, 14, 15, 16, 17, 18.

Bromus hordeaceus L., T, Subcosm, 1, 2, 3, 6, 8, 9, 10, 12, 14, 17, 18.

Bromus madritensis L., T, Med, 1, 2, 3, 4, 5, 6, 7, 8, 9, 10, 11, 13, 14, 15, 16, 17, 18.

Bromus rigidus Roth, T, Med, 1, 2, 3, 4, 5, 6, 7, 8, 9, 10, 12, 14, 16, 17.

Bromus rubens L., T, Med, 1, 3, 4, 5, 7, 8, 9, 13, 14.

Bromus sterilis L., T, Eurosib, 1, 2, 3, 5, 6, 7, 10, 14, 15, 16, 17, 18.

Bromus tectorum L., T, Plur, 3.

Dactylis glomerata L., H, Eurosib, 1, 2, 3, 4, 6, 7, 8, 9, 10, 13, 14, 15, 16, 18.

Desmazeria rigida (L.) Tutin subsp. rigida, T, Med, 8 .

Echinaria capitata (L.) Desf., T, Med, 3.

Elymus hispidus (Opiz) Melderis, G, Plur, 1, 2, 3, 4, 7, 10, 14, 15, 18.

Elymus pungens (Pers.) Melderis, G, Med W, 12, 15, 17.

Elymus repens (L.) Gould, G, Subcosm, 11, 12, 13.

Festuca gr. ovina L., H, Plur, 4, 6, 10.

Holcus lanatus L., H, Plur, 11, 12.

Hordeum distichon L., T, Neof, 1, 2, 3, 5, 6, 8, 9, 12, 14, 16.

Hordeum murinum L., T, Plur, 1, 2, 3, 4, 5, 6, 7, 8, 9, 10, 11, 12, 13, 14, 15, 16, $17,18$.

Koeleria vallesiana (Honckeny) Gaudin, H, Med W, 4, 10, 11, 14, 16.

Lolium rigidum Gaudin subsp. rigidum, T, Plur, 1, 2, 3, 4, 5, 6, 8, 9, 10, 12, 14, 16, 17, 18.

Lophochloa cristata (L.) Hyl., T, Subcosm, 1, 2, 3, 17.

Melica ciliata subsp. magnolii (Gren. \& Godr.) Husnot, H, Med W, 11, 13, 14.

Phalaris canariensis L., T, Neof, 6.

Phleum pratense subsp. bertolonii (DC.) Bornm., H, Plur, 4.

Piptatherum miliaceum (L.) Coss., H, Med, 1.

Poa annua L., T, Subcosm, 3, 9, 12, 14, 17.

Sclerochloa dura Beauv., T, Med, 6.

Secale cereale L., T, Neof, 5.

Setaria verticillata (L.) Beauv., T, Subcosm, 6.

Stipa barbata Desf., H, Med, 4.

Stipa capillata L., H, Eurosib, 4, 10.

Stipa pennata subsp. eriocaulis (Borbás) Martinovsky \& Skalicky, H, Eurosib, 11.

Triticum turgidum L., T, Neof, 1, 5, 6, 7, 8, 9, 14.

Vulpia ciliata Dumort. subsp. ciliata, T, Med, 1, 3, 5, 7, 9, 11, 12, 13, 14, 17.

Vulpia unilateralis (L.) Stace, T, Med, 4, 16.

Zea mays L., T, Neof, 6.

\section{Guttiferae}

Hypericum perforatum L. subsp. perforatum, H, Subcosm, 1, 14.

\section{Labiatae}

Ajuga chamepitys (L.) Schreb. subsp. chamaepitys, T, Med, 1.

Ballota nigra subsp. foetida Hayek, C, Plur, 5, 8, 9, 10, 15. 
Lamium amplexicaule L. subsp. amplexicaule, T, Plur, 2, 3, 5, 7, 8, 9, 10, 12, 15.

Lavandula latifolia Medik., C, Med, 10.

Marrubium vulgare L., C, Plur, 1, 2, 3, 4, 5, 6, 8, 9, 10, 11, 13, 14, 16, 17, 18.

Phlomis herba-venti L., H, Med, 10, 13, 14, 15.

Phlomis lychnitis L., C, Med W, 4, 8, 9, 10, 14, 15.

Rosmarinus officinalis L., F, Med, 9.

Salvia aethiopis L., H, Plur, 5, 11, 14, 15, 18.

Salvia lavandulifolia Vahl, C, End-ib, 4, 10, 11.

Salvia verbenaca L., H, Med, 1, 4, 5, 6, 8, 9, 10, 13, 14, 15, 16, 18.

Sideritis hirsuta L., C, Med W, 9, 10.

Sideritis linearifolia Lam., C, Med W, 4, 10, 11.

Sideritis montana subsp. ebracteata (Asso) Murb., T, Med W, 10.

Sideritis spinulosa Barnades ex Asso, C, End-ib, 11.

Teucrium chamaedrys L., C, Med, 4, 8, 9, 10, 11, 14.

Teucrium polium subsp. capitatum (L.) Arcang., C, Med W, 1, 4, 9, 10, 11, 14.

Thymus mastichina L. subsp. mastichina, C, Ib-nort, 4.

Thymus mastigophorus Lacaita, C, End-ib, 4.

Thymus zygis L. subsp. zygis, C, Ib-nort, 4, 9, 10, 14.

\section{Leguminosae}

Anthyllis vulneraria subsp. gandogeri (Sagorski) W. Becker ex Maire, H, Ib-nort, 1, 9.

Astragalus cymbaecarpos Brot., T, Ib-nort, 1, 3, 14.

Astragalus hamosus L., T, Med, 1, 3, 8, 9.

Astragalus sesameus L., T, Med, 1.

Astragalus stella Gouan, T, Med W, 6.

Coronilla scorpioides (L.) W.D.J. Koch, T, Med, 1, 4, 18.

Dorycnium pentaphyllum Scop., C, Med W, 4, 7, 10, 11, 13, 16, 18.

Genista scorpius (L.) DC., F, Med W, 10, 11.

Hippocrepis commutata Pau, C, End-ib, 4, 9, 11.

Lens culinaris Medik., T, Neof, 6.

Lotus corniculatus L. subsp. corniculatus, T, Med, 12.

Medicago lupulina L., H, Plur, 1, 2, 5, 11.

Medicago minima (L.) L., T, Plur, 1, 2, 4, 14, 17, 18.

Medicago polymorpha L., T, Plur, 3.

Medicago rigidula (L.) All., T, Med, 1.

Medicago sativa L., H, Subcosm, 1, 2, 3, 4, 5, 6, 7, 8, 9, 10, 11, 12, 13, 14, 15, 16, 17, 18.

Melilotus albus Medik., H, Subcosm, 1, 3 .

Melilotus indicus (L.) All., T, Subcosm, 1, 4.

Melilotus officinalis (L.) Pallas, H, Subcosm, 1, 2, 3, 4, 5, 8, 9.

Melilotus sulcatus Desf., T, Med, 8, 9.

Ononis pusilla L. subsp. pusilla, C, Med, 4, 9.

Ononis spinosa L., C, Plur, 4, 6, 9, 11, 18.

Trifolium campestre Scherb., T, Plur, 1.

Trifolium scabrum L., T, Med, 1.

Trigonella monspeliaca L., T, Med, 1.

Trigonella polyceratia L., T, Med W, 1 .

Vicia angustifolia L., T, Plur, 5.

Vicia eriocarpa (Hausskn.) Halácsy, T, Med, 5, 9.

Vicia monantha subsp. calcarata (Desf.) Romero Zarco, T, Med W, 1.

Vicia sativa L. subsp. sativa, T, Subcosm, 9. 


\section{Liliaceae}

Allium sphaerocephalon L. subsp. sphaerocephalon, G, Plur, 4, 11 .

Ornithogalum narbonense L., 15.

\section{Linaceae}

Linum austriacum subps. collinum Nyman, H, Med, 1, 4, 10.

Linum narbonense L., C, Med, 8, 11 .

Linum strictum L. subsp. strictum, T, Med, 4, 10, 11, 18.

Linum suffruticosum L. subsp. suffruticosum, C, Med W, 4.

\section{Malvaceae}

Alcea rosea L., H, Neof, 15, 17.

Althaea hirsuta L., T, Med, 11.

Malva neglecta Wallr., T, Plur, 3, 5, 6, 7, 8, 9, 12, 14, 17.

Malva nicaeensis All., T, Plur, 1, 3, 6, 7, 12, 13, 14, 17, 18.

Malva parviflora L., T, Med, 16.

Malva sylvestris L., H, Plur, 1, 2, 3, 4, 5, 6, 7, 8, 9, 10, 14, 15, 16, 17.

\section{Onagraceae}

Epilobium hirsutum L., H, Plur, 12.

\section{Orobanchaceae}

Orobanche minor Sm., (sobre Eryngium campestre), T, Subcosm, 4.

\section{Papaveraceae}

\section{Fumaria densiflora DC., T, Med, 5.}

Fumaria officinalis subsp. wirtgenii (W.D.J. Koch) Arcang., T, Med W, 12, 18.

Fumaria parviflora Lam., T, Med, 3, 5, 7, 8, 9, 16.

Fumaria vaillantii Loisel., T, Eurosib, 12.

Papaver argemone L., T, Med, 3.

Papaver hybridum L., T, Med, 1, 2, 3, 12, 17.

Papaver rhoeas L., T, Plur, 1, 2, 3, 4, 5, 6, 7, 8, 9, 10, 11, 12, 13, 14, 15, 16, $17,18$.

Roemeria hybrida (L.) DC., T, Med, 8.

\section{Pinaceae}

Pinus halepensis Mill., F, Med, 11.

\section{Plantaginaceae}

Plantago albicans L.,C, Med, 10, 11.

Plantago coronopus L., H, Plur, 12, 17.

Plantago lanceolata L., H, Subcosm, 1, 2, 3, 4, 5, 6, 7, 8, 9, 10, 11, 12, 13, 14, 15, 16, $17,18$.

Plantago sempervirens Crantz, C, Med W, 11.

\section{Polygalaceae}

Polygala monspeliaca L., T, Med, 4.

\section{Polygonaceae}

Fallopia convolvulus (L.) A. Löve, T, Plur, 5.

Polygonum aviculare L., T, Subcosm, 1, 2, 3, 5, 6, 8, 12, 14, 16, 17, 18.

Polygonum bellardii All., T, Med, 9. 
Polygonum persicaria L., T, Subcosm, 11.

Rumex conglomeratus Murray, $\mathrm{H}$, Plur, 1, 2, 5, 7, 8 .

Rumex crispus L., H, Subcosm, 1, 3, 4, 6, 7, 8, 9, 10, 12, 13, 16, 17.

Rumex pulcher subsp. woodsii (De Not.) Arcang., H, Plur, 1, 2, 3, 4, 5, 6, 8, 9, 10, 12, 13, 14, 15, $16,17,18$.

\section{Portulacaceae}

Portulaca oleracea L., T, Subcosm, 6, 14, 17.

\section{Primulaceae}

Anagallis foemina Mill., T, Plur, 7, 13.

Anagallis monelli L., H, Med W, 1.

Asterolinon linum-stellatum (L.) Duby, T, Med, 4.

Coris monspeliensis L. subsp. monspeliensis, C, Med W, 11.

\section{Ranunculaceae}

Consolida ajacis (L.) Schur, T, Neof, 11.

Consolida orientalis (J. Gay) Schrödinger, T, Med, 9.

\section{Resedaceae}

Reseda lutea L. subsp. lutea, H, Plur, 1, 2, 3, 5, 6, 7, 8, 9, 10, 11, 12, 13, 14, 15, 18.

Reseda luteola L., H, Plur, 3, 15.

Reseda phyteuma L., H, Med, 4.

Reseda undata L. subsp. undata, H, End-ib, 8, 9, 15, 17.

\section{Rosaceae}

Prunus dulcis (Mill.) D.A. Webb, F, Neof, 1.

Rosa gr. canina L., F, Plur, 2, 4, 6, 9, 11, 14, 15.

Sanguisorba verrucosa (Link ex G. Don) Ces., H, Plur, 1, 4, 9, 11, 15.

\section{Rubiaceae}

Asperula aristata subsp. scabra (J. \& C. Presl) Nyman, 10, 11, 14.

Galium aparine L., T, Subcosm, 1, 2, 3, 4, 5, 6, 7, 8, 9, 12, 13, 15, 16, 17, 18.

Galium lucidum All., C, Med, 2, 6, 8 .

Galium parisiense L. subsp. parisiense, T, Med, 1, 2, 4, 9, 10, 11, 15.

Galium tricornutum Dandy, T, Plur, 5, 7, 9.

Galium verum L. subsp. verum, H, Eurosib, 7, 8, 9, 10, 11, 13, 14, 15.

Rubia tinctorum L., H, Neof, 4, 5, 8, 13, 18.

\section{Rutaceae}

Haplophyllum linifolium (L.) G. Don fil., C, Med W, 4.

Ruta montana (L.) L., 14.

\section{Salicaceae}

Populus nigra L., F, Plur, 1.

\section{Santalaceae}

Thesium humifusum DC., C, Med, 4. 


\section{Scrophulariaceae}

Bartsia trixago L., T, Med, 1, 4, 13, 14, 18.

Linaria simplex (Willd.) DC., T, Med, 1.

Verbascum pulverulentum Vill., H, Med, 1, 5, 11, 14, 15, 18.

Verbascum thapsus L., H, Eurosib, 1, 3, 5, 9, 14.

Veronica persica Poir., T, Subcosm, 18.

\section{Solanaceae}

Datura stramonium L., T, Neof, 1.

Hyoscyamus niger L., H, Plur, 1, 3, 4, 5, 6, 8, 9, 10, 12, 15, 16, 18.

Solanum nigrum L. subsp. nigrum, T, Subcosm, 1, 5.

Solanum tuberosum L., G, Neof, 6, 8, 16.

\section{Umbelliferae}

Ammi majus L., T, Med, 1.

Ammi visnaga (L.) Lam., T, Med, 11.

Anthriscus caucalis M. Bieb., T, Plur, 2, 3, 13, 15.

Bupleurum baldense Turra, T, Med, 4.

Caucalis platycarpos L., T, Med, 9.

Conium maculatum L., H, Plur, 1, 2, 3, 5, 6, 7, 8, 9, 10, 14, 15.

Daucus carota L. subsp. carota, H, Subcosm, 1, 2, 3, 4, 6, 8, 9, 11, 14, 15, 18.

Eryngium campestre L., H, Plur, 1, 2, 3, 4, 5, 6, 7, 8, 9, 10, 11, 12, 13, 14, 15, 16, 17, 18.

Foeniculum vulgare Mill., H, Plur, 1, 2, 5, 6, 7, 8, 10, 11, 12, 13, 16, 17.

Thapsia villosa L., H, Med W, 4, 8, 9, 10, 13.

Torilis arvensis (Huds.) Link, T, Med, 12, 14.

Torilis leptophylla (L.) Rchb. fil., T, Med, 4.

Torilis nodosa (L.) Gaertn., T, Plur, 1, 2, 3, 6, 9, 10, 14.

Trinia glauca (L.) Dumort, H, Med, 4, 10.

\section{Urticaceae}

Urtica dioica L., H, Subcosm, 10.

Urtica urens L., T, Subcosm, 3, 5, 9, 10, 11, 12, 13, 15, 16, 17, 18.

\section{Valerianaceae}

Centranthus calcitrapae (L.) Dufresne, T, Med, 4, 9, 10, 13.

\section{Zygophyllaceae}

Tribulus terrestris L., T, Subcosm, 17. 